\title{
Estrategias de Afrontamiento y Ansiedad-Depresión en Pacientes Diagnosticados con VIH/Sida
}

\author{
Copying Strategies, Anxiety and Depression in HIV/Aids Patients
}

\author{
Ana Milena Gaviria ${ }^{1}$, Japcy Margarita Quiceno ${ }^{1}$, Stefano Vinaccia ${ }^{1}$, \\ Universidad de San Buenaventura, Medellín, Colombia \\ Luz Adriana Martínez, Martha Cecilia Otalvaro \\ Universidad Pontificia Bolivariana, Medellín, Colombia \\ (Rec: 18 de octubre 2007 Acep: 26 de marzo 2009)
}

\begin{abstract}
Resumen
El objetivo de este estudio fue evaluar las estrategias afrontamiento y la ansiedad-depresión en 92 pacientes de ambos géneros diagnosticados con VIH/Sida en tratamiento antirretroviral de la ciudad de Medellín, Colombia. La investigación tuvo como diseño un estudio descriptivo transversal correlacional mediante encuesta. Para medir las estrategias de afrontamiento se utilizó la Escala de Estrategias de Coping Modificada (EEC-M) de 98 ítems y la ansiedad-depresión se midió a través de los cuestionarios de Zung (SAS y SDS). Los resultados evidenciaron muy bajos niveles de ansiedad y depresión y alto uso de estrategias de afrontamiento de tipo cognitivo (focalizadas en el problema), de búsqueda de apoyo en la religión y profesionales de la salud, alto empleo de la estrategia de control emocional y muy bajo uso de la estrategia de reacción agresiva. En conclusión, las estrategias focalizadas en la cognición como búsqueda de alternativas, refrenar afrontamiento, reevaluación positiva e incluso la religión y búsqueda de apoyo profesional que son fundamentales para encontrar soluciones a nivel cognitivo ante los síntomas físicos y reacciones emociones tuvieron más relevancia en los pacientes con VIH/Sida.
\end{abstract}

Palabras clave: Estrategias de afrontamiento, ansiedad-depresión, VHI/Sida.

\begin{abstract}
The aim of this study was to evaluate the copying strategies and depression-anxiety in 92 patients of both genders with a diagnosis of HIV/Aids from the city of Medellín, Colombia. The investigation was a descriptive transversal correlational study. To measure copying strategies the Copying Strategies Scale Modified (CSS-M) was used of 98 items and anxiety-depression was measured through the questionnaires of Zung (SAS y SDS). The results evidenced very low levels of anxiety and depression and high use of copying strategies of cognitive type (focalized in the problem), of support search in religion and health professionals, high employment of emotional control strategies and very low use of the strategy of aggressive reaction. In conclusion, the strategies focalized in the cognition like search of alternative (problem solving), to refrain copying, positive reevaluation and even the religion and search of support professional that are fundamental to find solutions at the cognitive level before the physical symptoms and emotional reactions had more relevance in the patients with HIV/Aids.
\end{abstract}

Key words: Copying strategies, depression-anxiety, HIV/Aids.

\footnotetext{
1 Grupo de Investigación Salud Comportamental. Universidad de San Buenaventura de Medellín, Colombia. Carrera 56C, No. 51-90, Apartado Aéreo 81240. Correos electrónicos: japcyps@hotmail.com; amigago@hotmail.com; vinalpi47@hotmail.com
} 


\section{Introducción}

Las enfermedades infecciosas son el grupo de afecciones que mayor impacto ha tenido en la historia de la humanidad y que aún constituyen el principal motivo a nivel sanitario. Un informe de la OMS resalta que en el año 1995 las infecciones aún constituían la principal causa de muertes prematuras en el mundo, agrupando casi un tercio del total de fallecimientos ( $17 \%$ de un total de 52 millones), más de la mitad de los cuales ( 9 de los 17 millones) afectaban a niños. De otro lado, la mitad de los habitantes del mundo continúan expuestos a infecciones endémicas de diversa índole como el VIH/Sida en los últimos 25 años (Arrizabalaga, 2000).

El VIH es la sigla del Virus de Inmunodeficiencia Humana. Se trata de un microorganismo que afecta el sistema inmunológico del ser humano hasta destruirlo. El virus actúa atacando los linfocitos CD4 (una especie de glóbulos blancos), que detectan y destruyen estos agentes para que la persona no desarrolle enfermedades. Por otro lado, el SIDA es la sigla del Síndrome de Inmunodeficiencia Adquirida, que es la consecuencia de la infección por la transmisión del VIH cuando se complica con una enfermedad seria, véase el sarcoma de Kaposi, un tipo raro de cáncer de piel, la neumonía por Pneumocystis Carinii (conocida comúnmente por sus siglas en inglés: PCP), (Vera, López, Ariza, Díaz, Flórez, Franco, et al., 2004). Las vías principales de transmisión son a través de los líquidos sexuales, la sangre y en el embarazo y el parto. Algunas personas pueden presentar síntomas como fiebre, dolor de cabeza, ganglios inflamados, cansancio, coyunturas y músculos doloridos, dolor de garganta durante las seis semanas de haberse infectado con VIH, sin embargo, la mayoría de las personas con VIH no tiene síntomas por años, más cuando se contrae una enfermedad y se tiene el SIDA los síntomas son más severos como fiebre que dura más de un mes, perdida de peso, cansancio extremo, diarrea por más de un mes, ganglios linfáticos agrandados, falta de claridad al pensar, mareos, entre otros (OMS, 2003; De Cobo-Martínez, 2003).

La pandemia del SIDA es un problema de gran magnitud que afecta al mundo y de forma más acusada a algunas regiones como, por ejemplo, África subsahariana, Europa oriental y América Latina (Bermúdez \& Teva, 2003, 2004). En Colombia se han reportado 40 mil casos de SIDA con una prevalencia del $0.7 \%$, indicando que las personas afectadas podrían llegar a $180 \mathrm{mil}$. De esta cifra (40 mil casos) un 18 por ciento corresponde al sexo femenino. Los estudios estiman, además, que hacia 2010 la prevalencia de la enfermedad, al ritmo actual, estaría llegando al 1.5\%, afectando aproximadamente entre 600 mil y 800 mil personas. En la actualidad la enfermedad del SIDA se da en un $51 \%$ entre la población heterosexual, $28 \%$ en bisexuales y $35 \%$ en homosexuales y otros. El $50 \%$ de los casos se ha adquirido a los 20 años de edad en promedio (Ministerio de Protección Social, 2004).
Como es de observarse a nivel epidemiológico la mayoría de las personas pueden desarrollar alguna incapacidad o enfermedad crónica (Simón, 1999). Entendiéndose por enfermedad crónica aquel trastorno orgánico-funcional que obliga a una modificación en el estilo de vida de un individuo y que es probable persista por largo tiempo (Sandín, 1999). En el caso de las personas con VIH/Sida, con la llegada de los tratamientos antirretrovirales altamente activos (HAART en inglés: Highly Active Anti-Retroviral Therapy) se ha demostrado su capacidad para suprimir la carga viral hasta niveles indetectables, incrementar los indicadores de funcionamiento inmunitario -tales como recuento de linfocitos CD4-, mejorar el estado clínico y disminuir la mortalidad. Estos resultados han hecho posible comenzar a hablar del VIH/Sida como una enfermedad crónica y compleja que implica un proceso multifactorial desde un modelo biopsicosocial (Vinaccia, 2003). En las personas con VIH no sólo se ve afectado su funcionamiento fisiológico, sino también su estado emocional y mental, ya que se ven enfrentados a una serie de estresores socioculturales, económicos y psicológicos que deben lidiar (Vinaccia, 2003; Carrobles, Remor \& Rodríguez-Alzamora, 2003).

El diagnóstico de enfermedad crónica cambia la forma como la persona se ve a sí misma y a su vida, afectando profundamente el autoconcepto y la adaptación dependerá de una multiplicidad de factores, como la edad, el género, el estatus social, el apoyo, la educación, las creencias religiosas, los patrones de personalidad, la inteligencia, los autoesquemas, los estilos de afrontamiento, el equilibrio o control emocional, etc. Igualmente, éstas aparecen, se mantienen o se agravan en función de las conductas individuales (Pérez, 1999); por lo tanto, es fundamental evaluar el concepto de estilos de afrontamiento para entender las estrategias que pone en acción o en marcha una persona cuando se enfrenta a una enfermedad como el VIH/Sida.

Lazarus (2000) define el afrontamiento como "aquellos esfuerzos cognitivos y conductuales constantemente cambiantes que se desarrollan para manejar las demandas específicas externas y/o internas que son evaluadas como excedentes o desbordantes de los recursos del individuo". Los autores han propuesto tres tipos de evaluación cognitiva: evaluación primaria, evaluación secundaria y reevaluación. En la primera solamente las situaciones evaluadas como estresantes son las que implican cambio. Éstas pueden ser de tres tipos: de daño o pérdida, de amenaza y de desafío. Daño o pérdida se refiere a aquellos casos en que un individuo ha recibido un perjuicio (por ejemplo, la pérdida de un ser querido o la presencia de una enfermedad crónica); la amenaza está relacionada con aquellos casos en los que se prevén daños o pérdidas, aunque éstos no han ocurrido y se pueden tratar de solucionarlos o afrontarlos de manera anticipada, y el desafío implica la revisión anticipada de situaciones de daño o pérdida, como la amenaza, pero a diferencia de ésta se acompaña de la valoración de que existen 
fuerzas o habilidades suficientes para afrontarlas con éxito y obtener ganancias. La evaluación secundaria se refiere al análisis que el sujeto hace de las conductas, los recursos o las habilidades de las cuales se dispone para enfrentar la situación. Cuando en la evaluación encuentra que no los hay o que son insuficientes, se desencadena la respuesta de estrés (psicofisiológica y emocional), además de las categorías de evaluación expuestas. Por eso es fundamental considerar otras dos clases: la percepción de anticipación y la percepción de control de la situación. Este último se refiere a que el sujeto descubra que una situación depende de él y la puede manejar con los recursos que dispone. La anticipación, por su parte, es prepararse para un suceso, junto con su carácter positivo o negativo, deseable o no deseable. Estos dos factores son fundamentales en el estrés, puesto que se ha encontrado que los sucesos percibidos como incontrolables o impredecibles son los que tienen impacto sobre la salud (Sandín, 1999; Quiceno, Vinaccia, Lozano, Castaño \& Fernández, en prensa). Finalmente en la selección de las respuestas, el organismo selecciona las posibles a las demandas percibidas y decide cuáles debe utilizar.

Las experiencias emocionales pueden influir en la salud física mediante sus consecuencias fisiológicas, existiendo ya muchas evidencias de que los estados emocionales negativos pueden prolongar las infecciones y retardar la cicatrización de heridas (Kiecolt-Glaser, McGuire, Robles \& Glaser, 2002), así como hacer al individuo más vulnerable a diversas enfermedades (Salovey, Rothman, Detweiler \& Steward, 2000).

Las experiencias emocionales son una de las fuentes de información que tienen las personas acerca de sí mismas, y además el estado emocional es uno de los factores que determina la accesibilidad de una información o pensamiento, por lo cual un estado de ánimo positivo o negativo hará más accesibles pensamientos positivos o negativos, respectivamente. Debido a esto, la evaluación que hace un individuo de su estado de salud puede variar dependiendo de cuál sea su estado anímico, lo que lleva a preguntar si el estado emocional influye en el reconocimiento o interpretación de síntomas físicos, y específicamente si las personas que exhiben un estado anímico negativo están más predispuestas a interpretar un indicio fisiológico como un posible problema de salud, aspecto sumamente relevante en las personas con VIH. Estos antecedentes tienen una importancia mayor, pues los eventos estresantes, luego de un diagnóstico de VIH y de todo el curso de la infección, pueden influir de forma más negativa en las personas con afecto negativo (estado y rasgo), o ser mejor tolerados y afrontados por las que tienen una afectividad positiva como rasgo de personalidad. Empíricamente, se ha observado que las personas, desde el momento en que reciben el diagnóstico -o incluso desde la toma de muestras para el examen diagnóstico-, experimentan en menor o mayor grado algún tipo de alteración emocional, con severidad, tiempo e intensidad variables (Villa \& Vinaccia, 2006; Barra, 2003, 2004).

En tal perspectiva, múltiples investigaciones realizadas en la última década muestran cómo el estrés, el afrontamiento y los estados emocionales influyen en el curso del SIDA. $\mathrm{Al}$ respecto, hay evidencia de que la depresión y el estrés producen cambios en el sistema nervioso, inmunológico y endocrino, lo que influye para el desencadenamiento y evolución de la enfermedad (Tobón \& Vinaccia, 2003).

Edo y Ballester (2006), en España, desarrollaron un estudio para conocer el estado emocional y la conducta de enfermedad en personas con la infección por VIH en comparación con pacientes oncológicos y población general. La muestra utilizada fue de 180 sujetos agrupados en: 63 pacientes seropositivos que eran asintomáticos, sintomáticos y enfermos de SIDA, el grupo oncológico fue de 57 pacientes que padecían diferentes tipos de cáncer y 60 sujetos conformaron el grupo control de personas sanas. Los instrumentos aplicados evaluaron ansiedad rasgo-estado (STAI), depresión (BDI), autoestima (RSEI), conducta anormal de enfermedad (IBQ), percepción del apoyo social (PAS-VIH y PAS-ONC) y grado de interferencia de la enfermedad (Escala de Adaptación). Los resultados indicaron que los pacientes seropositivos mostraron un perfil psicológico con un mayor grado de ansiedad estado-rasgo y niveles de depresión significativamente más altos que los otros grupos, así como una autoestima significativamente menor. De otro lado, estos pacientes manifestaron mayor preocupación por su salud, mayor grado de interferencia de la enfermedad en sus vidas y percepción de apoyo social significativamente menor que los pacientes oncológicos y población normal.

Arrivillaga, Correa, Varela, Holguín y Tovar (2006) desarrollaron en la ciudad de Cali, Colombia, una investigación con 47 mujeres diagnosticadas de VIH/Sida cuyo propósito fue la descripción y correlación de las variables psicológicas ansiedad, depresión, Percepción de Control sobre la Salud -PCS- y Percepción de Control sobre Eventos Estresantes de la Vida -PCEEV-. Como medidas se utilizaron la entrevista de datos sociodemográficos, características biomédicas y psicosociales en mujeres diagnosticadas con VIH/Sida (diseñada por los autores) y se aplicaron los cuestionarios HAD (ansiedad-depresión), PCS y el PCEEV. Los resultados indicaron bajos niveles de ansiedad y depresión, y niveles moderados en Percepción de Control sobre la Salud y Percepción de Control sobre Eventos Estresantes de la Vida. Las variables ansiedad y depresión presentaron una correlación positiva, así como la PCEEV con ansiedad y depresión y de otro lado se presentaron correlaciones negativas de depresión y ansiedad con PCS.

En un estudio desarrollado en los Estados Unidos por Coleman, Holzemer, Eller, Corless, Reynolds, Nokes, et al., (2006) el objetivo fue analizar la relación entre género y la oración como estrategia de afrontamiento para 
sobrellevar la sintomatología relacionada con el VIH (fatiga, la náusea, la depresión y la ansiedad) en una muestra de 448 afroamericanos seropositivos. Encontraron que no había diferencias de género en el uso de la oración como estrategia de afrontamiento ante la ansiedad. De otro lado la oración como estrategia de afrontamiento fue utilizada por más del 50\% de los participantes y tuvo alto impacto en los síntomas de fatiga, nauseas y depresión. Las diferencias en el uso de la oración de acuerdo al género fueron: las mujeres utilizan con mayor frecuencia la oración para afrontar la fatiga y los hombres la emplean para manejar los síntomas de nauseas y depresión.

Olley, Gxamza, Seedat, Theron, Taljaard, Reid, et al., (2003), llevaron a cabo un estudio en Sur África con el propósito de comparar la comorbilidad psiquiátrica, las estrategias de afrontamiento y la incapacidad en 44 hombres y 105 mujeres diagnosticados recientemente con el VIH/ Sida. Utilizaron como instrumentos el MINI International Neuropsychiatric Interview (MINI), el Carver Brief COPE y el Sheehan Disability Scale, además fueron evaluados comportamientos de riesgo. De acuerdo a los resultados se encontró que un $56 \%$ de los pacientes fueron diagnosticados con un trastorno psiquiátrico, como depresión mayor (34.9\%), trastorno distímico $(21.5 \%)$, trastorno de estrés postraumático (14.8\%) y dependencia al alcohol (10.1\%). No hubo ninguna diferencia de acuerdo al género en la incidencia de los trastornos del estado emocional en la muestra. Sin embargo, los hombres cubrieron los criterios diagnósticos para el abuso del alcohol o la dependencia, y participar en ciertos comportamientos sexuales peligrosos. Las mujeres presentaron mayor probabilidad en presentar el trastorno de estrés postraumático y como estrategias de afrontamiento utilizaron la planificación y la religión para sobrellevar la enfermedad. De otro lado, tampoco hubo diferencias de género importantes en la incapacidad.

Eller, Corless, Bunch, Kemppainen, Holzemer, Nokes, et al., (2005), en California llevaron a cabo un estudio con el objetivo de evaluar la experiencia de síntomas depresivos, las estrategias de autocuidado ante esos síntomas, respuesta de los síntomas ante las estrategias y el origen de aprendizaje de cada estrategia en 422 personas con VIH positivo. Los síntomas depresivos fueron comunes bajo el diagnóstico y tratamiento en las personas con VIH. Estos síntomas fueron asociados a la adhesión al tratamiento, a los comportamientos de riesgo y resultados desfavorables en la salud. Se evaluaron los síntomas depresivos a través de 80 palabras y frases que fueron agrupadas en 8 categorías: la inanidad, la tristeza, la soledad/aislamiento, la fatiga, el miedo/felicidad, la falta de la motivación, ideas suicidas y otros. De 111 estrategias de autocuidado fueron divididas en seis categorías: prácticas de terapias alternativas y complementarias, uso de técnicas de distracción, uso de antidepresivos, realización de actividad física y evitación y negación emocional. Las estrategias basadas en recursos de información fueron: ensayo y error (31\%), profesionales sanitarios $(28 \%)$, familia y amigos $(20 \%)$, clases/lectura $(8 \%)$, clero $(8 \%)$, grupos de apoyo $(4 \%)$ y otro (3\%). Alrededor de un $92 \%$ de las estrategias fueron reportadas como efectivas. En conclusión, las personas con VIH utilizan numerosas estrategias de autocuidado para manejar los síntomas de depresión.

En una investigación realizada en tres países por Kemppainen, Eller, Bunch, Hamilton, Dole, Holzemer, et al., (2006) tuvieron como objetivo examinar la frecuencia y la eficacia de las estrategias de afrontamiento más utilizadas en el manejo de la ansiedad en una muestra internacional de 502 participantes seropositivos de Noruega $(n=42)$, Taiwán $(n=35)$ y los EE.UU. $(n=425)$. Todos los sujetos respondieron a un checklist de cinco categorías asociadas a comportamientos de autocuidado en salud como: actividades/pensamientos, ejercicio físico, medicación, terapias complementarias y uso de sustancias determinadas para la salud. En una escala ordinal de 1 a 10 fueron encontradas como estrategias de afrontamiento más utilizadas: rezar $(8.10 \%)$ como la más alta, seguida de la meditación (7.37\%), el ejercicio $(7.32 \%)$, las técnicas de relajación (7.22\%), cocinar (6.98\%) y caminar (6.90\%). De acuerdo a un análisis de las estrategias de afrontamiento desarrolladas en cada país para manejar la ansiedad hubo variaciones. En Noruega las estrategias de afrontamiento más utilizadas fueron: ejercicio $(7.31 \%)$ caminar $(6.96 \%)$ y leer $(6.44 \%)$. En Taiwán las estrategias más utilizadas fueron: hablar con otros pacientes con VIH (6.0\%), participar en grupos de apoyo $(6.0 \%)$ y realizar ejercicios $(6.0 \%)$. En los Estados unidos las estrategias más usadas fueron: las terapias complementarias y alternativas incluyendo la oración $(8.10 \%)$, la meditación $(7.43 \%)$ y el uso de técnicas de relajación $(7.35 \%)$. Sin considerar el país, ver la televisión y hablar con familia y amigos eran las dos estrategias más frecuentemente informadas.

Quiceno, et, al., (en prensa) en un estudio desarrollado en Colombia tuvieron como objetivo identificar la relación existente entre estrategias de afrontamiento y estrés en un grupo de personas con VIH/Sida de la ciudad de Medellín. Participaron 50 pacientes diagnosticados con Síndrome de Inmunodeficiencia Adquirida SIDA (9 mujeres y 41 hombres), cuyas edades oscilaron entre los 19 y 60 años, a quienes se les aplicaron los Cuestionarios: Estrategias de Afrontamiento de Coping y Sucesos Vitales CVS. A nivel general, el estudio evidenció que estos pacientes manifiestan un alto nivel de estrés frente a sucesos vitales como trabajo, matrimonio/pareja, hijos y finanzas, y las estrategias de afrontamiento más utilizadas fueron Solución de problemas, Refrenar afrontamiento, Expresión emocional, Religión, Búsqueda de apoyo profesional, Control emocional y Evitación comportamental.

A partir de lo anterior se planteó como objetivo general de esta investigación descriptivo transversal correlacional 
Tabla 1.Características sociodemográficas de los participantes del estudio.

\begin{tabular}{|c|c|c|c|c|c|}
\hline & & Frec. $(\%)$ & & & Frec. $(\%)$ \\
\hline \multirow{2}{*}{ Género } & Hombre & $78(84.8 \%)$ & \multirow{5}{*}{ Estado civil } & Soltero & $71(77.2 \%)$ \\
\hline & Mujer & $14(15.2 \%)$ & & Casado & $9(9.8 \%)$ \\
\hline \multirow{5}{*}{$\begin{array}{l}\text { Estrato } \\
\text { socioeconómico }\end{array}$} & 1 & $8(8.7 \%)$ & & Separado & $6(6.5 \%)$ \\
\hline & 2 & $21(22.8 \%)$ & & Viudo & $1(1.1 \%)$ \\
\hline & 3 & $38(41.3 \%)$ & & Unión Libre & $5(5.4 \%)$ \\
\hline & 4 & $18(19.6 \%)$ & \multirow{3}{*}{ Escolaridad } & Primaria & $11(12.0 \%)$ \\
\hline & 5 & $7(7.6 \%)$ & & Secundaria & $38(41.3 \%)$ \\
\hline \multirow{5}{*}{ Ocupación } & Desempleado & $18(19.6 \%)$ & & Superior & $43(46.7 \%)$ \\
\hline & Empleado Informal & $12(13.0 \%)$ & \multirow{4}{*}{$\begin{array}{l}\text { Tiempo de } \\
\text { diagnóstico }\end{array}$} & De 0 a 12 meses & $3(3.3 \%)$ \\
\hline & Empleado & $49(53.3 \%)$ & & De 12 a 24 meses & $15(16.3 \%)$ \\
\hline & Estudiante & $3(3.3 \%)$ & & Más de 24 meses & $74(80.4 \%)$ \\
\hline & Otros & $10(10.9 \%)$ & & & \\
\hline
\end{tabular}

tipo encuesta (Montero \& León, 2002) evaluar las estrategias afrontamiento y la ansiedad-depresión en pacientes de ambos géneros diagnosticados con VIH/Sida en tratamiento antirretroviral de la ciudad de Medellín, Colombia. Como objetivos específicos se consideró, en primer lugar, medir los niveles de ansiedad y depresión bajo los criterios de la escala HAD, en segundo lugar medir las estrategias de afrontamiento a través de la Escala de Estrategias de Coping Modificada (EEC-M) de 98 ítems y en tercer lugar analizar la relación de las variables del estudio entre sí.

\section{Método}

\section{Participantes}

Participaron en este estudio 92 pacientes voluntarios entre los 23 a 68 años con una media de edad de 39.4, de ambos géneros, con diagnóstico de VIH/Sida en tratamiento antirretroviral en una Entidad Prestadora de Servicios de Salud (EPS) de la ciudad de Medellín, Colombia. En la Tabla 1 se presentan las características sociodemográficas de la muestra: Se observa que la mayor parte de los participantes fueron hombres (84.8\%) y sólo un $15.2 \%$ fueron mujeres. En relación al estrato socioeconómico, un porcentaje mayor (64.1\%) se agrupa en el nivel 2 y 3 (medio-bajo). En cuanto al nivel educativo, la mayoría de los sujetos tiene estudios superiores $(46.7 \%)$, seguido de estudios básicos en secundaria $(41.3 \%)$ y primaria $(12.0 \%)$. A nivel de la variable ocupación, se observó que la mayoría realiza una actividad laboral ya sea formal e informal $(66.3 \%)$ y el resto se ubicó en las categorías desempleado (19.6\%), estudiante (3.3\%) y otros (10.9\%). Respecto al estado civil, alrededor de una tercera parte de la muestra $(77.2 \%)$ está ubicada en la categoría de solteros. Por último, en los pacientes con VIH/Sida se encontró que tenían más de dos años de tiempo de diagnóstico de enfermedad.

\section{Instrumentos}

Se utilizó la Escala de Estrategias de Coping Modificada (EEC-M) validada en Colombia por Londoño, Henao, Puerta, Posada, Arango y Aguirre (2006) y desarrollada originalmente por Chorot y Sandín (1993). La escala EEC-M de tipo likert tiene una frecuencia de respuesta que va de 1 (Nunca), 2 (Casi nunca), 3 (A veces), 4 (Frecuentemente) 5 (Casi siempre) hasta 6 (Siempre). Está compuesta por 98 ítems agrupados en catorce factores, cada uno con siete ítems y el rango para cada factor oscila de 7 a 42 puntos. La escala EEC-M comprende los siguientes factores: 1 . Búsqueda de alternativas (antes Focalización en la situación problema), 2. Conformismo (antes Autofocalización negativa), 3. Control Emocional (antes Autocontrol), 4. Evitación Emocional, 5. Evitación Comportamental (antes Evitación), 6. Evitación Cognitiva (antes Evitación), 7. Reacción Agresiva (Irritabilidad) (antes Expresión emocional abierta), 8. Expresión emocional abierta, 9. Reevaluación positiva (antes Reestructuración Cognitiva), 10. Búsqueda de apoyo social (conserva su nombre), 11. Búsqueda de apoyo profesional (conserva su nombre), 12. Religión (conserva su nombre), 13. Refrenar el afrontamiento (adicionada), y 14. Espera. El alfa de Cronbach de la escala total fue de 0.84 .

Para evaluar la ansiedad y la depresión se utilizaron las escalas autoaplicadas de Zung:

Para medir la ansiedad se utilizó la escala autoadministrada de Zung (1971), Self-rating Anxiety Scale (SAS), con índice de Cronbach superior a 0.6 en la escala total. Esta escala está compuesta por 20 ítems, donde 5 ítems hacen referencia a síntomas afectivos y 15 ítems a síntomas somáticos de ansiedad. Consta de un formato tipo likert, con una escala de frecuencia de cuatro puntos que van de 1 (ausente), 2 (ligera), 3 (Moderada) hasta 4 (Fuerte). La escala da puntuaciones entre 25 y 100 puntos. Las puntuaciones de 50 o más sugieren síntomas de ansiedad con 
importancia clínica. Esta escala no ha sido formalmente validada en Colombia, no obstante ha sido ampliamente utilizada (Pareja \& Campo, 2006).

Para evaluar la depresión se utilizó la Escala Autoaplicada de Depresión de Zung (1965), Self-Rating Depression Scale (SDS). Es una escala de cuantificación de síntomas de base empírica y derivada de la escala de depresión de Hamilton, ya que al igual que ella da mayor peso al componente somático-conductual del trastorno depresivo. Fue validada al español originalmente por Conde, Escribá, Izquierdo (1970), con un índice de Cronbach entre 0.79 y 0.92. Esta escala ha sido utilizada ampliamente en Colombia, véase entre otras Campo, Díaz, Rueda y Barros (2005); Arrivillaga, Cortés, Goicochea y Lozano (2004). La versión española tipo likert está compuesta por 20 ítems con una escala de 4 puntos, desde 1 (raramente o nunca) hasta 4 (casi todo el tiempo o siempre). Contiene además los siguientes puntos de corte: No depresión $<35$ ( $<28$ puntos), Depresión leve 36-51 (28-41 puntos); Depresión moderada 52-67 (42-53 puntos), Depresión grave $>68$ ( $>53$ puntos).

\section{Procedimiento}

Previo permiso y autorización de la institución Prestadora de Servicios de Salud (EPS) de la ciudad de Medellín, Colombia y disponibilidad a la base de datos, se contactaron a los pacientes diagnosticados con VIH/Sida a través de vía telefónica y de acuerdo a la disponibilidad de éstos se les asignaba una cita para diligenciar las pruebas. Éstos fueron reunidos en diferentes días de la semana por grupos de cinco personas, máximo siete, en las instalaciones de la EPS en un periodo de dos meses y medio. Los pacientes, quienes acudían a consulta externa, fueron informados acerca de los objetivos de la investigación y una vez brindada la información, se solicitó a éstos el consentimiento para participar en el estudio, comentándoles acerca del secreto ético de la información y que podrían retirarse de la investigación cuando ellos lo consideraran. Una vez obtenido el consentimiento informado, se diligenció la ficha de datos sociodemográficos y se les entregó los tres instrumentos (EEC-M, SAS y SDS), los cuales fueron autodiligenciados por los pacientes con la supervisión del entrevistador cuando éstos la requerían, con una duración aproximada de una hora.

\section{Resultados}

Se observa en la tabla 2 las diferentes medidas de tendencia central como lo son la media, la moda, la Desviación Típica, los puntajes mínimos y máximos, y los Alfa de Cronbach de cada uno de los instrumentos psicométricos y sus diferentes dimensiones. A nivel general todo el estudio tuvo un índice de confiabilidad superior a .68 .

A nivel general se observa que los pacientes del estudio tuvieron puntuaciones muy bajas en las escalas de Zung

Tabla 2. Estadísticas descriptivas de las variables del estudio: Estrategias de Afrontamiento, Ansiedad y Depresión.

\begin{tabular}{lcccccc}
\hline & Media & Moda & D.T. & Mínimo & Máximo & Alfa de Cronbach \\
\hline Escala EEC-M & & & & & & \\
Búsqueda de alternativas & 30.9 & 39 & 7.5 & 15 & 44 & 0.68 \\
Conformismo & 21.9 & 25 & 5.6 & 9 & 37 & 0.67 \\
Control emocional & 29.6 & 29 & 5.4 & 15 & 41 & 0.66 \\
Evitación emocional & 25.5 & 22 & 7.3 & 9 & 42 & 0.66 \\
Evitación comportamental & 24.1 & 17 & 6.2 & 11 & 39 & 0.64 \\
Evitación cognitiva & 24.6 & 23 & 7.7 & 9 & 42 & 0.62 \\
Reacción agresiva & 14.8 & 11 & 6.4 & 7 & 37 & 0.70 \\
Expresión emocional abierta & 23.6 & 23 & 5.1 & 12 & 35 & 0.68 \\
Reevaluación positiva & 28.5 & 28 & 7.4 & 13 & 42 & 0.66 \\
Búsqueda de apoyo social & 22.6 & 17 & 7.6 & 9 & 40 & 0.69 \\
Búsqueda de apoyo profesional & 28.2 & 34 & 8.4 & 12 & 42 & 0.68 \\
Religión & 29.8 & 42 & 9.7 & 7 & 42 & 0.66 \\
Refrenar el afrontamiento & 29.7 & 29 & 6.5 & 14 & 42 & 0.65 \\
Espera & 19.7 & 16 & 6.6 & 8 & 39 & 0.66 \\
Escala de Zung SAS & & & & & & 0.66 \\
Ansiedad & 34.1 & 26 & 9.8 & 20 & & \\
Escala de Zung SDS & & & & & 54 & 0.69 \\
Depresión & 41.7 & 35 & 6.3 & 29 & 54 & \\
\hline
\end{tabular}


Figura 1. Puntaciones medias de la Escala de Estrategias de Coping Modificada (EEC-M) de una muestra poblacional sana y los pacientes con VIH/Sida.

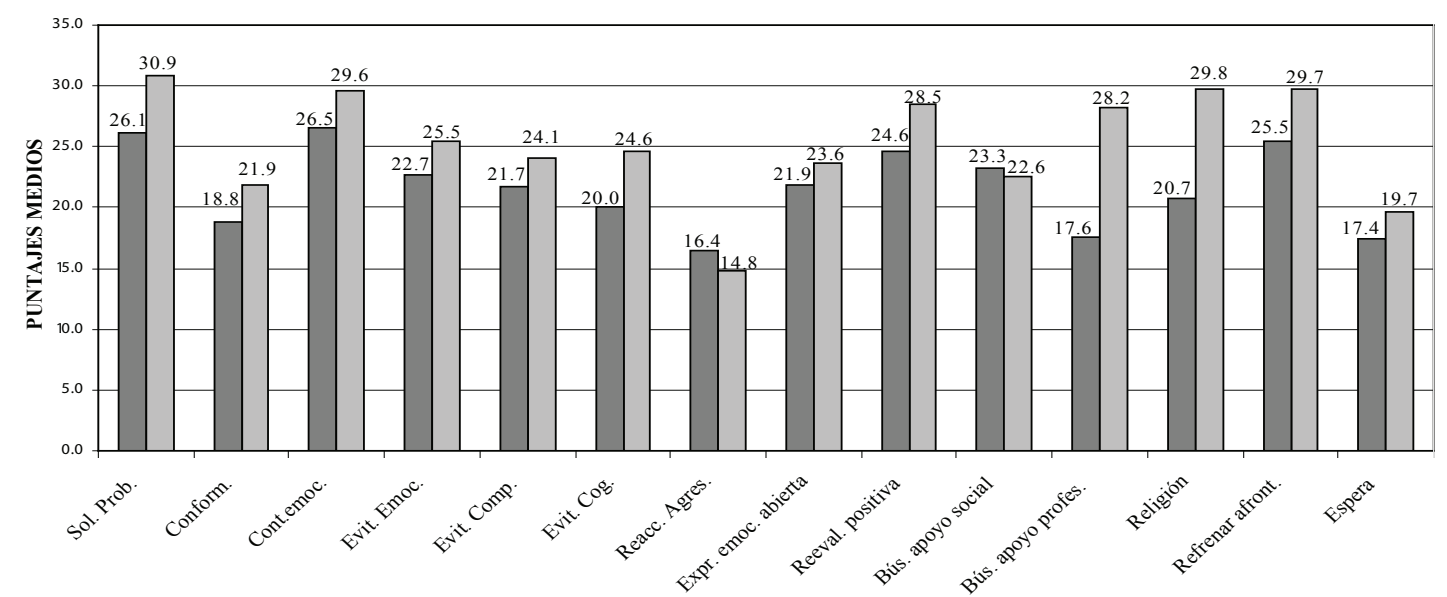

ES TRATEGIAS DE AFRONTAMIENTO

$\square$ Muestra Sana $\square$ Pacientes VIH/SIDA

Tabla 3. Correlación de las diferentes estrategias de afrontamiento de la Escala EEC-M

\begin{tabular}{|c|c|c|c|c|}
\hline & Evitación cognitiva & Reevaluación positiva & Refrenar el afrontamiento & Espera \\
\hline Búsqueda de alternativas & & $*_{0.66}$ & $*_{0.77}$ & \\
\hline Conformismo & & & & $*_{0.71}$ \\
\hline Control emocional & & $*_{0,60}$ & & \\
\hline Evitación emocional & $*_{0.62}$ & & & \\
\hline Reevaluación positiva & & & $*_{0.65}$ & \\
\hline
\end{tabular}

de ansiedad y depresión, los cuales están por debajo de los puntos de corte de las investigaciones tanto españolas como colombianas desarrolladas en el área, indicando que los sujetos con VIH/Sida de nuestro estudio no presentaron sintomatología ansioso-depresiva.

En cuanto a las estrategias de afrontamiento más utilizadas por los pacientes de nuestra investigación medidas por la Escala EEC-M se encontraron puntuaciones altas en las estrategias de Búsqueda de alternativas, Religión, Refrenar el afrontamiento, Reevaluación positiva y Búsqueda de apoyo profesional, siendo las más significativas Búsqueda de alternativas, Religión y Refrenar el afrontamiento de toda la escala. De otro lado, la estrategia de afrontamiento Reacción agresiva tuvo la puntuación más baja de toda la Escala EEC-M. En una puntuación media alta se encontró la estrategia de Control emocional y en un puntaje medio bajo la estrategia de Espera. El resto de las estrategias se ubicaron en niveles dentro del promedio como: Conformismo, Evitación emocional, Evitación comportamental, Evitación cognitiva, búsqueda de apoyo social y Expresión emocional abierta.
A manera de información, se presenta en la Figura 1 la comparación de los puntajes medios de la Escala de Estrategias de Coping Modificada (EEC-M) de 98 ítems, validada en Colombia por Londoño, et. al., (2006) con una muestra poblacional sana de la ciudad de Medellín y los pacientes con VIH/Sida de nuestro estudio. Se observa a nivel general que los puntajes de las estrategias de afrontamiento más usadas por los sujetos de nuestra investigación son mucho más elevados que los de la muestra de personas sanas, siendo la más significativa búsqueda de apoyo profesional y religión, seguida de solución de problemas, evitación cognitiva, reevaluación positiva y refrenar afrontamiento. De otro lado, se encuentran diferencias en las estrategias de reacción agresiva y búsqueda de apoyo social que tuvieron puntajes un poco más bajos en los pacientes de nuestro estudio en comparación con la muestra sana. Indicando que las estrategias de tipo cognitivo, de búsqueda de información, de apoyo espiritual y control emocional, son las más empleadas en los pacientes con VIH/Sida de nuestro estudio. 
En cuanto a los resultados de la aplicación del Coeficiente de Correlación de Pearson a todas las variables del estudio, encontramos que hubo correlaciones altas positivas $(p>0.5)$ entre algunas de las estrategias de afrontamiento de la Escala EEC-M entre sí, especialmente de aquellas de tipo cognitivo, donde lo cognitivo tiene alta relación con lo emocional y viceversa, como se observa en la tabla 3.

\section{Discusión}

En el presente estudio se trabajó con una muestra de 92 pacientes, mayoritariamente hombres, adultos jóvenes, solteros, de estrato socioeconómico medio-bajo, con un nivel educativo superior, laboralmente activos y con dos años de tiempo de diagnóstico de la enfermedad. Los resultados de nuestro estudio en cuanto a género y edad son muy similares al informe reportado por el Ministerio de Protección Social (2005), donde se plantea que en Colombia un $80 \%$ de la población con diagnóstico de VIH/Sida es masculina con edades entre los 25 a 34 años.

En nuestro estudio los pacientes con VIH/Sida no presentaron niveles clínicamente significativos de ansiedad y depresión. Igualmente en otros estudios desarrollados en Colombia se han encontrado resultados similares en enfermos crónicos, tanto en VIH/Sida (Arrivillaga, et. al., 2006) como en otras enfermedades crónicas, véase enfermedad pulmonar obstructiva crónica, lupus eritematoso sistémico, esclerosis múltiple, síndrome del colon irritable, entre otras enfermedades estudiadas por el mismo grupo de investigación (Vinaccia, Quiceno, Zapata, Obesso \& Quintero, 2006; Vinaccia, Quiceno, Zapata, Abad, Pineda \& Anaya, 2006a; Vinaccia, Quiceno, Zapata, Gonzáles \& Villegas, 2006b; Vinaccia, Contreras, Fernández, Amador, Tamayo, Vásquez, et. al., 2005).

Los estudios antes mencionados con muestras poblacionales colombianas difieren significativamente con otras investigaciones desarrolladas en distintas partes del mundo donde se ha encontrado altos niveles de ansiedad y depresión en pacientes con VIH/Sida (Coleman, et al., 2006; Olley, et al., 2003; Eller, et. al., 2005; Kemppainen, et al., 2006; Edo \& Ballester, 2006).

Los datos colombianos que reportan bajos niveles de ansiedad y depresión en pacientes con VIH/Sida y en enfermedades crónicas son en cierto sentido paradójicos en comparación con los estudios epidemiológicos recientes sobre salud mental con población general colombiana donde se han encontrado altos niveles de ansiedad y depresión (Gómez, Bohórquez, Pinto, Gil, Rondón \& Díaz-Granados, 2004; Ministerio de Protección Social, 2007).

Por otro lado, en nuestra muestra de pacientes con VIH/ Sida se encontró alto uso de estrategias de afrontamiento de tipo cognitivo (focalizadas en el problema), de búsqueda de apoyo en la religión y profesionales de la salud, viéndose reflejado en la baja capacidad de espera. Igualmente se encontró alto empleo de la estrategia de control emocional viéndose reflejado en la baja agresividad. Las estrategias focalizadas en la cognición como búsqueda de alternativas, refrenar afrontamiento, reevaluación positiva e incluso la religión y búsqueda de apoyo profesional que son fundamentales para encontrar soluciones cognitivas ante los síntomas físicos y reacciones emociones tuvieron más relevancia en los pacientes con VIH/Sida que las estrategias focalizadas en la emoción. Lo anterior indica que hubo supresión de pensamientos negativos sustituyéndolos por frecuentes pensamientos positivos que les favorecieran tolerar, planificar o tomar alternativas viables para buscar soluciones ante la enfermedad del VIH/Sida. Igualmente el alto control emocional posiblemente tenga relación con las estrategias cognitivas, ya que la alta racionalidad ante el mismo comportamiento emotivo-conductual puede haber neutralizado los sentimientos de ira/hostilidad. Todo lo anterior puede verse corroborado en el análisis correlacional del estudio y en las puntaciones medias presentadas en el gráfico 1 de la Escala de Estrategias de Coping Modificada (EEC-M) de 98 ítems, validada en Colombia por Londoño, et al., (2006) con una muestra poblacional sana de la ciudad de Medellín y los pacientes con VIH/Sida de nuestro estudio.

Los datos de nuestro estudio son similares al estudio desarrollado por Quiceno et al., (en prensa) en Colombia y se asemeja a otras investigaciones desarrollados en otras partes del mundo en relación a las estrategias de afrontamiento de religión expresada a través de la oración, el rezo y la meditación y búsqueda de apoyo a través de la familia, amigos, los grupos de apoyo y profesionales sanitarios (Coleman, e. al., 2006; Olley, et al., 2003; Eller, et al., 2005; Kemppainen, et al., 2006). Sin embargo, puede observarse en relación a estos autores que aunque hayan estrategias de afrontamiento más comúnmente utilizadas por los pacientes de $\mathrm{VIH} / \mathrm{Sida}$, existen otras estrategias más específicas y relacionadas con determinados contextos socioculturales, como por ejemplo: prácticas de terapia alternativas, técnicas de relajación, ejercicios físicos, uso de medicamentos psiquiátricos u otros medicamentos, la lectura, ver televisión, cocinar y caminar.

En conclusión, los factores psicosociales y sociodemográficos como la edad, el género, el modelo cultural y educativo, la ocupación, el estado civil, la percepción de apoyo social, las creencias relacionadas con la enfermedad, los autoesquemas, el locus de control externo e interno hacia la salud, los rasgos de personalidad, las habilidades sociales, los estilos de afrontamiento, las emociones negativas, las enfermedades crónicas asociadas, las enfermedades psiquiátricas o psicológicas antes y después del diagnóstico de la enfermedad, los efectos secundarios de los tratamientos médicos y farmacológicos utilizados, entre otras, pueden en algunos casos, permitir o no a los pacientes con VIH/ Sida afrontar la enfermedad para lograr la adaptación y el bienestar de su salud general. 


\section{Referencias}

Arrivillaga, M., Correa, D., Varela, M., Holguín, L. \& Tovar, J. (2006). Variables psicológicas en mujeres diagnosticadas con VIH/SIDA: un estudio correlacional. Universitas Psychologica, 5, 659-667.

Arrivillaga, M., Cortés, C., Goicochea, V. \& Lozano, T. (2004). Caracterización de la depresión en jóvenes universitarios. Universitas Psychologica, $3,17-25$.

Arrizabalaga, J. (2000). Las «enfermedades emergentes» en las postrimerías del siglo XX: El sida. Política y Sociedad, 35, 93-100.

Barra, E. (2004). Apoyo social estrés y salud. Psicología y Salud, 14, 237-243.

Barra, E. (2003). Influencia del Estado Emocional en la Salud Física. Terapia Psicológica, 21, 55-60.

Bermúdez, M.P \& Teva, I. (2004). Situación actual del SIDA en España: análisis de las diferencias entre comunidades autónomas. International Journal of Clinical and Health Psychology, 4, 553-570.

Bermúdez, M.P \& Teva, I. (2003). Situación actual del VIH/SIDA en Europa: análisis de las diferencias entre países. International Journal of Clinical and Health Psychology, 3, 89-106.

Campo, A., Díaz, L.A., Rueda, G.E. \& Barros, J.A. (2005). Validación de la escala de Zung para depresión en universitarias de Bucaramanga, Colombia. Revista Colombiana de Psiquiatría, 34, 54-62.

Carrobles, J.A., Remor, E. \& Rodríguez-Alzamora, L. (2003). Afrontamiento, apoyo social percibido y distrés emocional en pacientes con infección por VIH. Psicothema, 15, 420-426.

Coleman, C.L., Holzemer, W.L., Eller, L.S., Corless, I., Reynolds, N., Nokes, K.M., Kemppainen, J.K., Dole, P., Kirksey, K., Seficik, L., Nicholas, P. \& Hamilton, M.J. (2006). Gender differences in use of prayer as a self-care strategy for managing symptoms in African Americans living with HIV/ AIDS. Journal Association Nurses AIDS Care, 17, 16-23.

Conde, V., Escribá, J.A. \& Izquierdo, J. (1970). Evaluación estadística y adaptación castellana de la escala autoaplicada para la depresión de Zung. Archivos de Neurobiología, 33,185-206.

De Cobo-Martínez, F. (2003). Aspectos clínicos y microbiológicos de la infección por VIH: nuevos avances en el tratamiento del SIDA (2 ED). Jaén: Alcala la Real

Edo, M.T. \& Ballester, R. (2006). Estado emocional y conducta de enfermedad en pacientes con VIH/SIDA y enfermos oncológicos. Revista de Psicopatología y Psicología Clínica, 11, 79-90.

Eller, L.S., Corless, I., Bunch, E.H., Kemppainen, J., Holzemer, W., Nokes, K., Portillo, C. \& Nicholas, P. (2005). Self-care strategies for depressive symptoms in people with HIV disease. Journal Advance Nurses, 51, 119-130.

Gómez, C., Bohórquez, A., Pinto, D., Gil, J., Rondón, M. \& Díaz-Granados, N. (2004). Prevalencia de depresión y factores asociados con ella en la población colombiana. Revista Panamericana de Salud Pública, 16, 378-386.

Kemppainen, J.K., Eller, L.S., Bunch, E., Hamilton, M.J., Dole, P., Holzemer, W., Kirksey, K., Nicholas, P.K., Corless, I.B., Coleman, C., Nokes, K.M., Reynolds, N., Sefcik, L., Wantland, D. \& Tsai, Y.F. (2006). Strategies for self-management of HIV-related anxiety. AIDS Care, 18, 597-607.

Kiecolt-Glaser, J.K., McGuire, L., Robles, T. \& Glaser, R. (2002). Psychoneuroimmunology: Psychological influences on immune function and health. Journal of Consulting and Clinical Psychology, 70, 537-547.

Lazarus, R.S. (2000). Estrés y emoción: Manejo e implicaciones en nuestra salud. Bilbao : Desclée de Brouwer

Londoño, N.H., Henao, G.C., Puerta, I.C., Posada, S., Arango, D. \& Aguirre, D.C. (2006). Propiedades psicométricas y validación de la escala de estrategias de coping modificada (EEC-M) en una muestra colombiana. Universitas Psychologica, 5, 327-349.

Ministerio de Protección Social (2007). Estudio nacional de salud mental Colombia 2003. Ministerio de la Protección Social-OMS/Harvard-FES. En: Documentos y publicaciones. Extraído el 22 Febrero, 2007 del sitio Web Ministerio de Protección Social: http://www.minproteccionsocial. gov.co/VBeContent/home.asp y http://www.minproteccionsocial.gov. co/vbecontent/library/documents/DocNewsNo15417DocumentNo2663. PDF
Ministerio de Protección Social (2005). El 80\% de los casos de VIH/SIDA corresponde a hombres. En: Sala de prensa. Extraído el 22 Febrero, 2007 del sitio Web Ministerio de Protección Social: http://www.minproteccionsocial.gov.co/VBeContent/home.asp y

http://www.minproteccionsocial.gov.co/salaprensa/NewsDetail. asp?ID $=14260 \&$ IDCompany $=13$

Ministerio de Protección Social (2004). 40 mil casos reportados de SIDA en Colombia. En: Noticias SNE. Extraído el 12 Febrero, 2007 del sitio Web: de Presidencia de la República de Colombia: http://www.presidencia. gov.co/prensa_new/sne/ y http://www.presidencia.gov.co/prensa_new/ sne/2004/noviembre/29/12292004.htm

Montero, I. \& León, O. (2002). Clasificación y descripción de las metodologías de investigación en psicología. International Journal of Clinical and Health Psychology, 2, 503-508.

Olley, B.O., Gxamza, F., Seedat, S., Theron, H., Taljaard, J., Reid, E., Reuter, H. \& Stein, D.J. (2003). Psychopathology and coping in recently diagnosed HIV/AIDS patients--the role of gender. South Africa Medicine Journal, 93, 928-931.

Organización Mundial de la Salud (2003). Situación de la epidemia de SIDA. WHO: New York

Pareja, A. \& Campo, A. (2006). Prevalencia de síntomas de ansiedad en pacientes con cefalea primaria. Acta Neurológica Colombiana, 22, 299-303.

Pérez, J. (1999). Respuestas emocionales, enfermedad crónica y familia. En: Enrique Fernández \& Francesc Palmero (Editores). Emociones y Salud. Barcelona: Ariel

Quiceno, J.M., Vinaccia, S., Lozano, I.C., Castaño, Z. \& Fernández, H. (en prensa). Estrés y estrategias de afrontamiento en un grupo de personas con VIH. En: Juan José Sánchez-Sosa, Julio Alfonso Piña López y Blanca Margarita Rivera Icedo (Editores). Aportaciones de la psicología al problema de la infección por VIH: investigación e intervención. México: Universidad Nacional Autónoma de México.

Salovey, P., Rothman, A.J., Detweiler, J.B. \& Steward, W.T. (2000). Emotional states and physical health. American Psychologist, 55, 110-121.

Sandín, B. (1999). Estrés psicosocial. Madrid: Klinik.

Simón, M. (1999). Tratado de psicología de la salud. Madrid: Biblioteca Nueva; 1999.

Tobón, S. \& Vinaccia, S. (2003). Modelo de intervención psicológica en el VIH/sida. Psicología y Salud, 13, 161-174.

Vera, L.M., López, N., Ariza, N.D., Díaz, L.M., Flórez, Y., Franco, S.G., Isaza, S., Marciales, D.P., Ortiz, N., Rueda, J. \& Torres, L.J. (2004). Asociación entre el área de estudio y los conocimientos y comportamientos frente a la transmisión del VIH/SIDA en los estudiantes de la Universidad Industrial de Santander. Colombia Médica, 35, 62-68.

Villa, I.C \& Vinaccia, S. (2006). Adhesión terapéutica y variables psicológicas asociadas en pacientes con diagnóstico de VIH-sida. Psicología y Salud, 16, 51-62.

Vinaccia, S., Quiceno, J.M., Zapata, C., Obesso, S. \& Quintero, D.C. (2006). Calidad de vida relacionada con la salud y emociones negativas en pacientes con diagnóstico de Enfermedad pulmonar obstructiva crónica (EPOC). Psicología desde el Caribe.

Vinaccia, S., Quiceno, J.M., Zapata, C., Abad, L., Pineda, R. \& Anaya, JM. (2006a). Calidad de vida en pacientes con lupus eritematoso sistémico. Revista Colombiana de Psicología, 15, 57-65.

Vinaccia, S., Quiceno, J.M., Zapata, C., Gonzáles, A.C. \& Villegas, J. (2006b). Calidad de vida relacionada con la salud y emociones negativas en pacientes con diagnóstico de esclerosis múltiple. Revista Argentina de Clínica Psicológica, 15, 125-134.

Vinaccia, S., Contreras, F., Fernández, H., Amador, O., Tamayo, R., Vásquez, A., Tobón, S. \& Sandín, B. (2005). Evaluación de la calidad de vida, la ansiedad y depresión en pacientes con diagnóstico de síndrome de colon irritable. Terapia Psicológica, 23, 65-74.

Vinaccia, S. (2003). El patrón de conducta Tipo C en pacientes con enfermedades crónicas. Revista Colombiana de Psiquiatría, 32, 161-168.

Zung, W. (1971). A rating instrument for anxiety disorders. Psychosomatics, 12, 371-379.

Zung W. (1965). A self rating depression scale. Archivies General Psychiatry, $12,63-70$ 